\title{
Astrocytes protect glioma cells from chemotherapy and upregulate survival genes via gap junctional communication
}

\author{
QINGTANG LIN, ZHAO LIU, FENG LING and GENG XU \\ Department of Neurosurgery, Xuanwu Hospital, Capital Medical University, Beijing 100054, P.R. China
}

Received February 1, 2015; Accepted November 19, 2015

DOI: $10.3892 / \mathrm{mmr} .2015 .4680$

\begin{abstract}
Gliomas are the most common type of primary brain tumor. Using current standard treatment regimens, the prognosis of patients with gliomas remains poor, which is predominantly due to the resistance of glioma cells to chemotherapy. The organ microenvironment has been implicated in the pathogenesis and survival of tumor cells. Thus, the aim of the present study was to test the hypothesis that astrocytes (the housekeeping cells of the brain microenvironment) may protect glioma cells from chemotherapy and to investigate the underlying mechanism. Immunofluorescent and scanning electron microscopy demonstrated that glioma cells were surrounded and infiltrated by activated astrocytes. In vitro co-culture of glioma cells with astrocytes significantly reduced the cytotoxic effects on glioma cells caused by various chemotherapeutic agents, as demonstrated by fluorescein isothiocyanate-propidium iodide flow cytometry. Transwell experiments indicated that this protective effect was dependent on physical contact and the gap junctional communication (GJC) between astrocytes and glioma cells. Microarray expression profiling further revealed that astrocytes upregulated the expression levels of various critical survival genes in the glioma cells via GJC. The results of the present study indicated that the organ microenvironment may affect the biological behavior of tumor cells and suggest a novel mechanism of resistance in glioma cells, which may be of therapeutic relevance clinically.
\end{abstract}

Correspondence to: Dr Qingtang Lin or Professor Geng Xu, Department of Neurosurgery, Xuanwu Hospital, Capital Medical University, 45 Changchun Street, Xicheng, Beijing 100054, P.R. China

E-mail: kingsang2002@hotmail.com

E-mail: xugeng2006@sina.com

Abbreviations: $\mathrm{BBB}$, blood-brain-barrier; CBX, carbenoxolone; FACS, fluorescence-activated cell sorting; FITC, fluorescein isothiocyanate; 5-FU, 5-fluorouracil; GFAP, glial fibrillary acidic protein; GFP, green fluorescent protein; GJC, gap junctional communication; PI, propidium iodide; TMZ, temozolomide

Key words: astrocytes, glioma cells, protection, chemotherapy, gap junctional communication, survival genes

\section{Introduction}

Gliomas are the most common type of primary brain tumor, accounting for almost $40 \%$ of all brain neoplasms (1). They result from the transformation of glial cells, with astrocytes as the predominant glial type (2). The current treatment regimen for gliomas includes surgery, radiotherapy and chemotherapy (3). However, despite these treatment modalities, the prognosis for patients with glioma remains poor. The overall survival rate of patients with glioblastoma, the most malignant and frequent type of glioma, is extended only by $\sim 14.6$ months (4). This is predominantly due to the resistance of glioma cells to chemotherapy $(5,6)$. Traditionally, this resistance has been attributed to the blood-brain-barrier (BBB) $(7,8)$. However, clinical and laboratory studies have demonstrated that the BBB surrounding the gliomas permits leakage $(9,10)$. Therefore, the BBB is not likely to be the sole mechanism underlying this resistance.

The organ microenvironment has been implicated in the progression and survival of tumor cells $(11,12)$. Astrocytes are the most dominant cell type in the brain, which connect with each other through gap junctional communication (GJC) and, thus function as a syncicium (13). Under physiological conditions, astrocytes function as housekeeping cells and maintain homeostasis in the brain microenvironment (14-17). In pathological conditions, astrocytes become activated, via the upregulation of glial fibrillary acid protein (GFAP), and have been demonstrated to be capable of protecting neurons from various injuries, including trauma and ischemic insult (18-21). GJC, the molecular channel allowing the transmission of critical survival-associated secondary messengers, has been shown to be one of the mechanisms underlying this process $(22,23)$. Previous studies have suggested that there are activated astrocytes surrounding glioma cells, and that functional GJC exists between astrocytes and glioma cells $(24,25)$. These observations raise the possibility that reactive astrocytes may also protect glioma cells from chemotherapy with a mechanism, which may be associated with GJC. To confirm this hypothesis, the present study investigated the sensitivities of glioma cells treated with various chemotherapeutic drugs, in the presence or absence of astrocytes. The global gene expression patterns of glioma cells altered by the co-culture of astrocytes was also examined using microarray analysis. These investigations were performed to determine whether astrocytes protect glioma cells from the apoptosis induced by chemotherapeutic 
drugs, examine the expression levels of survival genes in glioma cells and determine the contribution of GJC between astrocytes and glioma cells. The findings of the present study provide an insight into resensitizing glioma cells toward chemotherapy, which may contribute to extending the limited survival time of patients with glioma.

\section{Materials and methods}

Cell lines, cell culture and reagents. The human glioma cell lines (U87MG, U251 and A172) and fibroblast cell line (NIH3T3) were purchased from the Cell Center of Peking Union Medical University (Beijing, China). Immortal mouse astrocytes were obtained from Dr Fidler's laboratory (University of Texas, MD Anderson Cancer Center, Houston, Texas, USA). All cell lines were recovered from frozen stocks and maintained in Dulbecco's modified Eagle medium supplemented with $10 \%$ fetal bovine serum, non-essential amino acids, L-glutamine, a two-fold vitamin solution (all from Thermo Fisher Scientific, Inc., Waltham, MA, USA), and a penicillin/streptomycin mixture (Flow Laboratories, Inc., Rockville, MD, USA). Cultures were maintained at $37^{\circ} \mathrm{C}$ in $5 \% \mathrm{CO}_{2}$, as previously described (26-28). Hochest 33342 and the GFAP antibody were purchased from Invitrogen (Thermo Fisher Scientific, Inc.). Carbenoxolone (CBX) and propidium iodide (PI) were purchased from Sigma-Aldrich (St. Louis, MO, USA). The temozolomide (TMZ), paclitaxel and 5-fluorouracil (5-FU) chemotherapeutic agents were purchased from Lunarsun Pharmaceuticals (Beijing, China). All reagents were dissolved in dimethyl sulfoxide (ABigen Corporation, Beijing, China), and reagents were of analytical reagent grade. A plasmid containing green fluorescent protein (GFP) was purchased from Abigen Corporation.

Animals. Five athymic Ncr-nu/nu male mice (age, 10 weeks; weight, 16-18 g) were purchased from the Animal Center of Peking Union Medical University (Beijing, China) and maintained under specific pathogen-free conditions with free access to food and water, at $20-25^{\circ} \mathrm{C}$ (humidity, $50 \pm 5 \%$ ), under a 12-h light/dark cycle. Animal protocols were approved and supervised by the Animal Care and Use Committee of Xuanwu Hospital affiliated with Capital Medical University (Beijing China) and were in accordance with institutional guidelines.

Glioma mouse model. The human glioma cells were harvested upon reaching 60-70\% confluence and were resuspended in phosphate-buffered saline (PBS; Abigen Corporation). 1x10 cells (volume, $5 \mu \mathrm{l}$ ) were introduced into the brain parenchyma of the mice via stereotactic injection, as described previously (29). Following the development of neurological symptoms in the mice, for example circling, the mice were sacrificed using $\mathrm{CO}_{2}$ and four brain tissue samples were harvested for analysis.

Scanning electron microscopy of the co-cultured glioma cells and astrocytes. The U87MG human glioma cells and murine astrocytes (1:1 ratio) were seeded onto sterilized glass coverslips in 24 -well plates, at a density of $2.4 \times 10^{4}$ cells/well. After $48 \mathrm{~h}$, the co-culture samples were processed as described previously (30). Briefly, the samples were fixed for $1 \mathrm{~h}$ at room temperature in a solution containing 3\% glutaraldehyde, $2 \%$ paraformaldehyde and $7.5 \%$ sucrose in $0.1 \mathrm{M}$ cacodylate buffer ( $\mathrm{pH} 7.3$; all Abigen Corporation). The samples were washed with $0.1 \mathrm{M}$ cacodylate buffer followed by distilled water and sequentially treated for $30 \mathrm{~min}$ in the dark with Millipore-filtered aqueous 1\% tannic acid (Merck Millipore, Darmstadt, Germany) and washed in distilled water and Millipore-filtered $1 \%$ aqueous uranyl acetate (Merck Millipore) for $1 \mathrm{~h}$ in the dark. Samples were mounted onto double-thick carbon tabs (Ted Pella, Inc., Redding, CA, USA). The samples were then coated under vacuum using a Balzer MED 010 evaporator (Technotrade International, Inc., Manchester, NH, USA) with platinum alloy to a thickness of $25 \mathrm{~nm}$ and immediately flash carbon coated under vacuum. Samples were examined using a JSM-5910 scanning electron microscope (JEOL USA, Inc., Peabody, MA, USA) at an accelerating voltage of $5 \mathrm{kV}$.

Assessment of chemoprotection using flow cytometric analysis. The GFP expressing astrocytes were established by transfection with the pLCMV vector containing a GFP expressing cassette dissolved in Lipofectamin 2000 (Invitrogen; Thermo Fisher Scientific, Inc.). Briefly, $4 \mu \mathrm{g}$ plasmid DNA and $10 \mu 1$ Lipofectamine 2000 were dissolved in $250 \mu \mathrm{l}$ transfection medium (Invitrogen Opti-MEM-I reduced serum medium; Thermo Fisher Scientific, Inc.) for $20 \mathrm{~min}$. The solution was combined further by mixing for another $5 \mathrm{~min}$ prior to being added into the culture medium. Eight hours after incubation, the medium was replaced with fresh antibody-free medium. Forty-eight hours later, the cells were harvested and GFP-positive cells were sorted via fluorescence-activated cell sorting (FACS; FACS Elite; BD Biosciences, Franklin Lakes, NJ, USA). The glioma cells (1x106; harvested at $60-70 \%$ confluence) were cultured in the presence or absence of GFP-expressing astrocytes at a ratio of 1:1 in the wells (diameter, $35 \mathrm{~mm}$ ) of the sterile, 6-well tissue culture plate. Following incubation for $72 \mathrm{~h}$ with the chemotherapeutic agents (TMZ, $5 \mu \mathrm{M}$; cisplatin, $1 \mu \mathrm{M}$; 5-FU, $2 \mu \mathrm{M})$ at $37^{\circ} \mathrm{C}$, the floating and adherent cells were collected and fixed with $70 \%$ ethanol. The PI staining procedure was performed, as described previously (31). Briefly, cells were harvested by trypsinization (Abigen Corporation) and washed once with PBS. Cells were centrifuged at 1,000 x g for $3 \mathrm{~min}$ at room temperature, and cell pellets were resuspended in $1 \%$ paraformaldehyde for $5 \mathrm{~min}$ to fix the GFP. Subsequently, the cells were centrifuged and resuspend in $70 \%$ ethanol and stored at $-20^{\circ} \mathrm{C}$ in a refrigerator overnight. At the day of analyzing, cells were centrifuged again and washed once with PBS. Cells were centrifuged and the PBS was removed; the resultant pellets were resuspended in PI staining buffer (containing $50 \mu \mathrm{g} / \mathrm{ml}$ PI in PBS, supplemented with 37.5 units of RNase enzyme; Abigen Corporation) and placed in an incubator at $37^{\circ} \mathrm{C}$ for $30 \mathrm{~min}$ prior to analysis. FACS analysis was performed using an EPICS XL cytometer (Beckman Coulter, Inc, Brea, CA, USA). The fluorescein isothiocyanate (FITC)-PI protocol was used to analyze the cell cycles of the GFP-expressing FITC-positive astrocyte population and the FITC-negative glioma cell population separately. Apoptotic cells were evaluated as the fraction of cells in the sub-G0 


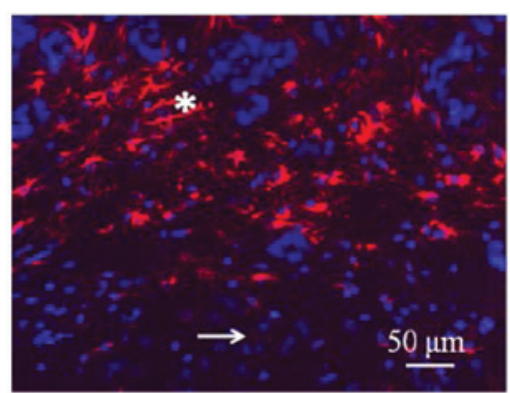

B

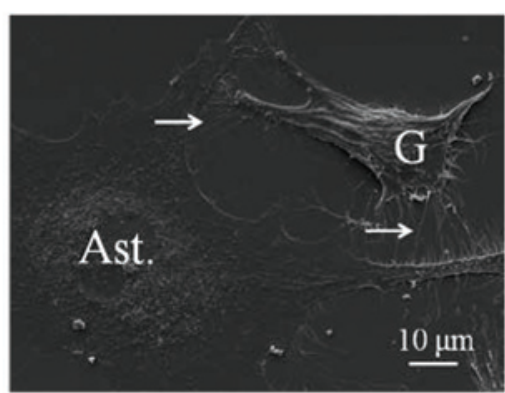

Figure 1. Interactions between astrocytes and glioma cells. (A) Immunofluorescent analysis of human U87MG glioma cells in the brain of athymic Ncr-nu/nu mice. In the tumor tissue (indicated by the asterisk), dividing glioma cells (Hochest33342-stained blue) were surrounded by activated astrocytes ( GFAP ${ }^{+}$-stained red). In the tumor-free tissue (indicated by the arrow), only sporadic GFAP (red) was expressed. (B) Scanning electron microscopy of astrocytes (Ast.) and U87MG glioma cells $(\mathrm{G})$ demonstrates the direct physical contacts between the astrocytes (extending end feet) and glioma cells, as indicated by the arrow. GFAP, glial fibrillary acid protein.

A

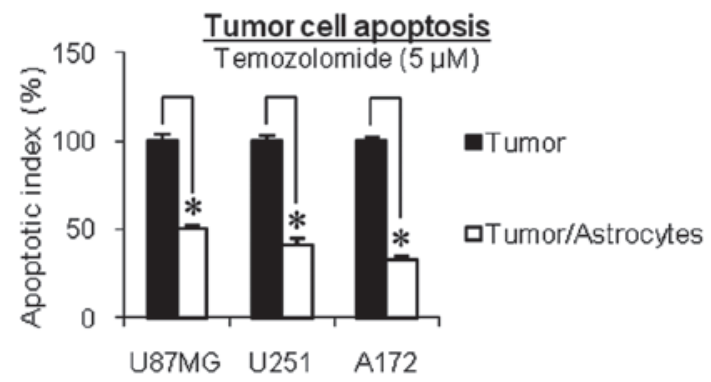

C

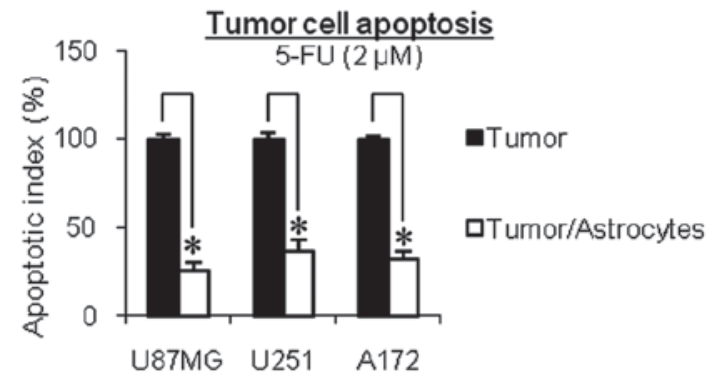

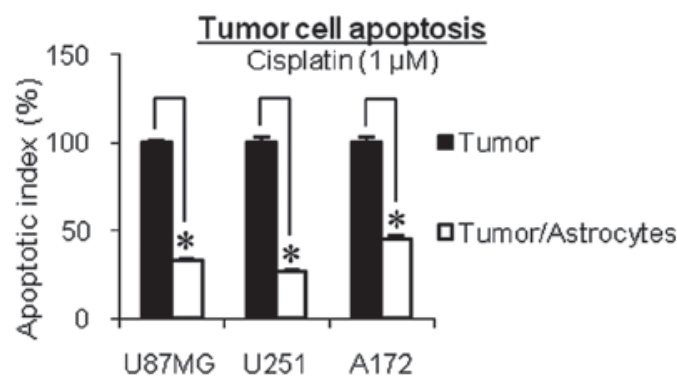

D

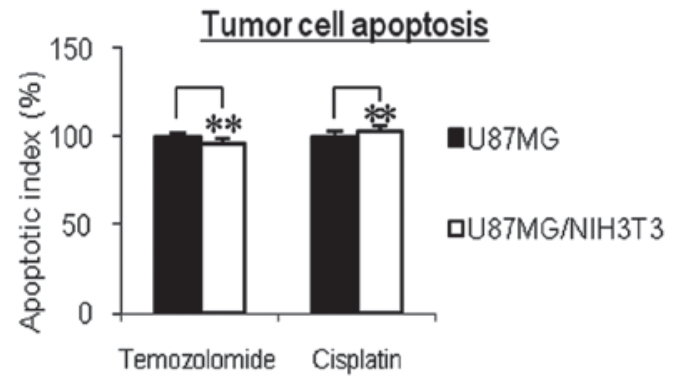

$\mathbf{E}$

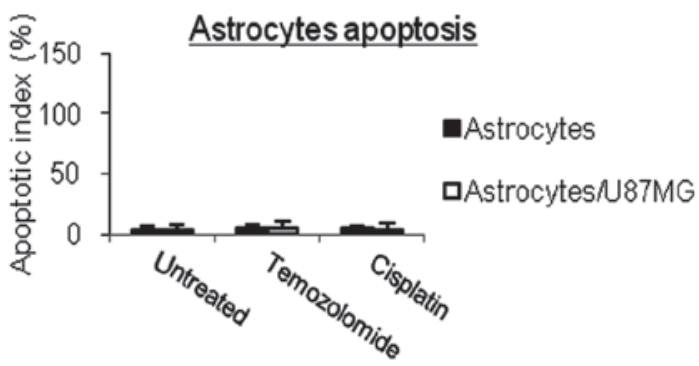

Figure 2. Astrocytes protect glioma cells from chemotherapeutic drugs. Co-culture with astrocytes resulted in a significant decrease in apoptosis in the glioma cells induced by (A) temozolomide, (B) cisplatin and (C) 5-FU. (D) Substituting NIH3T3 fibroblasts for astrocytes failed to demonstrated similar protective effects. (E) Astrocytes cultured alone or with glioma cells did not undergo apoptosis when treated with the same chemotherapeutic drugs. Data are expressed as the mean \pm standard error of the mean. ${ }^{*} \mathrm{P}<0.01 ;{ }^{* *} \mathrm{P}>0.05 .5-\mathrm{FU}, 5$-fluorouracil.

region. Levels of apoptosis in response to chemotherapeutic drugs in the glioma cells cultured alone or with astrocytes were assessed, as described above, for all the three glioma cell lines. To exclude the possibility of the protective effect being due to the co-culture of mouse cells with human cells, NIH3T3 mouse fibroblasts were co-cultured with U87MG glioma cells as a control.
Transwell experiment. To determine whether the protective effect is dependent on physical contact or mediated by secreted factors, a 6-well plate Boyden chamber (Costar Boyden Chamber; pore size, $0.4 \mu \mathrm{m}$; Corning Inc., Corning, NY, USA) was used in the present study. The transwell membrane pore size facilitates with the separation of tumor cells from astrocytes while allowing the passage of secreted 
A

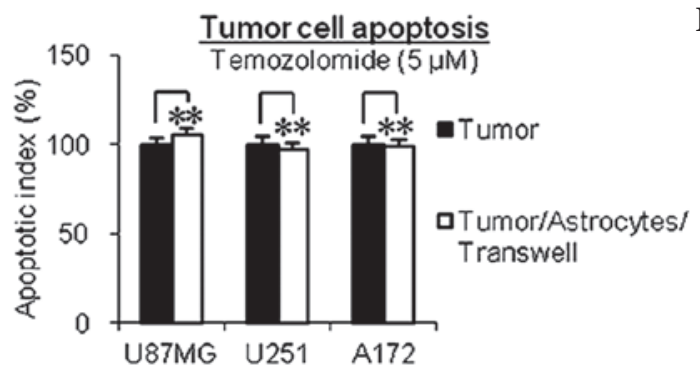

B

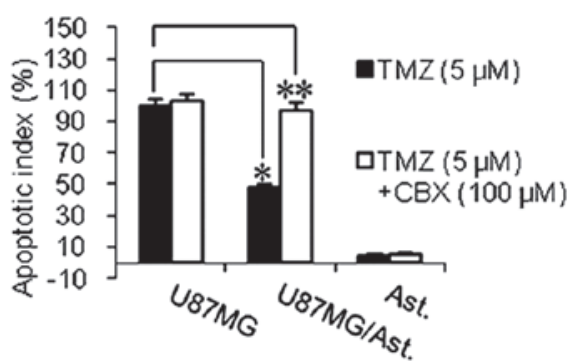

Figure 3. Protection depends on direct physical contact via GJC between astrocytes and glioma cells. (A) Separation of astrocytes from glioma cells by a semi-permeable Transwell membrane (0.4- $\mu \mathrm{m}$ pore size) eliminated the protective effects. (B) Application of CBX, a specific GJC inhibitor, completely reversed the protective effect. The addition of CBX to temozolomide did not affect apoptosis in the single cultures of astrocytes or U87MG glioma cells. Data are expressed as the mean \pm standard error of the mean. ${ }^{*} \mathrm{P}<0.01{ }^{* *} \mathrm{P}>0.05$. GJC, gap junctional communication; CBX, carbenoxolone; TMZ, temozolomide; Ast., astrocytes.

A

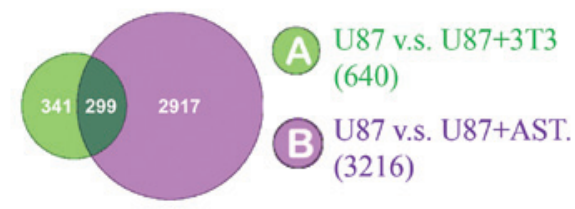

C

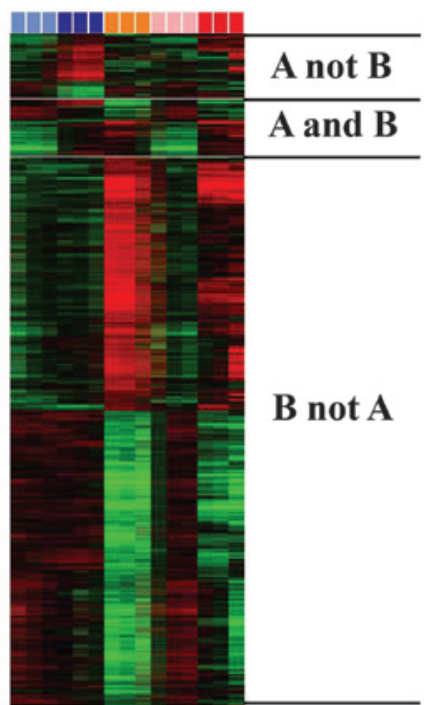

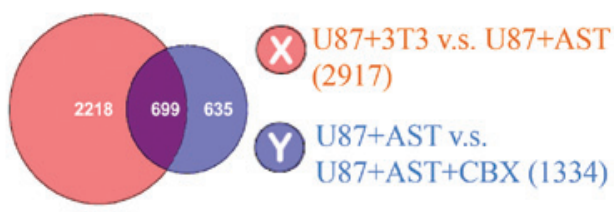

D

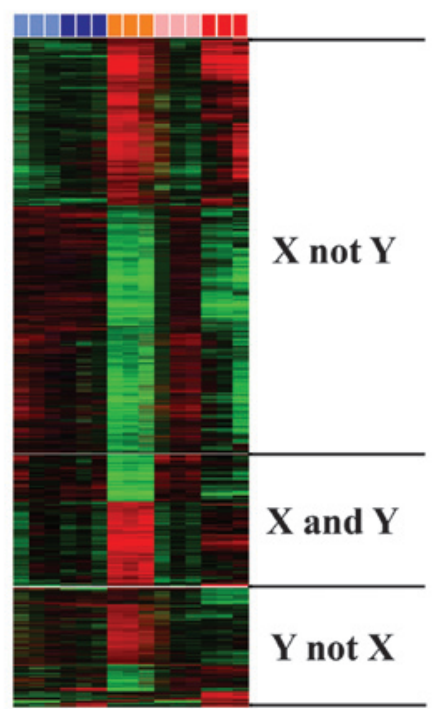

Figure 4. Gene expression patterns in U87MG glioma cells are altered by astrocytes via GJC. Cross comparison of gene lists from two independent statistical tests $(\mathrm{P}<0.001)$ was applied. A matrix format in heat map was used to present the data, in which rows represent individual genes and columns represent each tested hybridization event (in triplicate), with the intensity of color indicating the magnitude of expression (log2-transformed scale). Of note, different colors represent different cultures (light blue, U87MG alone; pink, U87MG with CBX; dark blue, U87MG with NIH3T3; orange, U87MG with astrocytes; red, U87MG with astrocytes and CBX). (A and B) Co-culture with astrocytes altered the expression of 3,216 genes in the U87MG glioma cells (group 'B'), whereas co-culture with NIH3T3 fibroblasts altered the expression of 640 genes in the glioma cells (group 'A'). The expression of 299 genes was altered by the two co-cultures, which were excluded from further analysis (group 'A and B'), with the remaining 2,917 genes regarded as the genes altered specifically by astrocytes (group 'B not A'). (C and D) Application of CBX altered the expression of 1,334 genes (group ' $\mathrm{Y}$ '), of which 699 genes were shared with the previous analysis (group ' $\mathrm{X}$ ') and thus regarded as genes that were altered by astrocytes through GJC. Several well-known genes associated with cell survival and drug resistance were among these 699 genes (group ' $\mathrm{X}$ and Y'). GJC, gap junctional communication; CBX, carbenoxolone; AST, astrocytes.

factors. Astrocytes ( $10^{5}$ cells) were seeded into the top insert of the Boyden chamber, and glioma cells $\left(10^{5}\right.$ cells $)$ were seeded in the bottom insert. Following overnight incubation at $37^{\circ} \mathrm{C}$, the chemotherapeutic agent (TMZ, $\left.5 \mu \mathrm{M}\right)$ was added to the medium. $72 \mathrm{~h}$ later, the inserts were removed and the cells (floating and adherent) were harvested and stained with PI, following the above-mentioned protocol. The cell cycle profiling was analyzed by FACS, with the sub- $\mathrm{G}_{0}$ and $-\mathrm{G}_{1}$ regions defined as the percentage of cell death.
Examination of gene expression profiles using RNA microarray analysis. The U87MG human glioma cells ( $10^{5}$ cells) were cultured alone, with murine astrocytes or with NIH3T3 fibroblasts in the $35 \mathrm{~mm}$-diameter 6-well plate (BD Falcon; BD Biosciences, San Jose, CA, USA), in the presence or absence of the GJC inhibitor, CBX $(100 \mu \mathrm{M})$. For the cell co-culture, the ratio of glioma cells to murine astrocytes or NIH3T3 cells was set as $1: 1$. Following incubation for $72 \mathrm{~h}$ at $37^{\circ} \mathrm{C}$, the GFP-labeled murine astrocytes and fibroblasts were sorted 
using FACS, and the glioma cells were harvested for microarray analyses. For microarray analyses, total RNA (500 ng) was collected for labeling and hybridization, in accordance with the manufacturer's protocol (Illumina, Inc, San Diego, CA, USA), with the use of Illumina's Sentrix human 6-v2 Expression BeadChips. The BeadChips were scanned using an Illumina BeadArray Reader (Illumina, Inc). The results of the microarray data were extracted using Bead Studio 3.7 (Illumina, Inc.) without any normalization or background subtraction. Normalization of gene expression data was performed using the quantile method in LIMMA package in R (www.rproject. org) (32). The expression level of each gene was transformed into a $\log 2$ value prior to further analysis. In order to select genes, which were differentially expressed in the two culture groups, a class comparison tool was used in the BRB array tools (v3.6; Biometrics Research Branch, National Cancer Institute, Bethesda, MD, USA), as a two-sample $t$-test with the estimation of false discovery rate. All experiments were performed in triplicate to avoid potential false positive genes due to technical variance.

Statistical analysis. All data are presented as the mean \pm standard error of the mean. Student's $t$-test was performed to compare the values between the different treatment groups. SPSS version 19 was used to perform statistical analyses (IBM SPSS, Armonk, NY, USA) and P<0.05 was considered to indicate a statistically significant difference.

\section{Results}

Interactions between astrocytes and glioma cells. It has been demonstrated previously that there are reactive astrocytes surrounding glioma cells in human specimen $(24,25)$ and, in the present study, similar observations were made (data not shown). In the glioma in situ xenograft animal model, as shown in Fig. 1A, the U87MG glioma cells were infiltrated and surrounded by GFAP-positive activated astrocytes (red fluorescence). In the in vitro co-culture conditions, astrocytes formed direct contact with the U87MG glioma cells, as shown by scanning electron microscopy in Fig. 1B. These direct contacts between the astrocytes and glioma cells were also evident in the in vivo conditions.

Astrocytes protect glioma cells from cytotoxicity caused by chemotherapeutic agents. Following co-culture with astrocytes, the apoptotic rates of the U87MG, U251 and A172 glioma cells induced by the TMZ $(5 \mu \mathrm{M})$ chemotherapeutic drug were significantly reduced, by $49.5 \pm 1.5 \%(\mathrm{P}<0.05)$, $58.8 \pm 4.2 \%(\mathrm{P}<0.05)$ and $67.1 \pm 1.6 \%(\mathrm{P}<0.05)$, respectively (Fig. 2A). This protection against the chemotherapeutic drug was not specific to TMZ, as the similar reductions in the apoptosis of U87MG, U251 and A172 glioma cells were observed in the astrocytes exposed to cisplatin $(66.6 \pm 1.4$, $73.2 \pm 0.7$ and $54.2 \pm 1.8 \%$, respectively; Fig. 2B) and 5-FU (74.5 $\pm 4.2,63.8 \pm 6.6$ and $68.7 \pm 5.3 \%$, respectively; Fig. $2 \mathrm{C}$ ). To rule out the possibility that this protection was due to the co-culture of human cells (glioma cells) with murine cells (astrocytes), murine NIH3T3 fibroblasts were included as a control. As shown in Fig. 2D, the murine NIH3T3 fibroblasts failed to protect glioma cells from the chemotherapeutic agents. The astrocytes, when cultured either alone or with glioma cells, did not undergo apoptosis when treated with the same chemotherapeutic drugs (Fig. 2E).

Protection of glioma cells by astrocytes is dependent on physical contact GJC. To investigate whether the protection provided by the astrocytes is dependent on direct physical contact or mediated by secreted factors, the present study used a semi-permeable Transwell membrane (Transwell-Boyden Chamber; 0.4- $\mu \mathrm{m}$ pore size membrane), which ensures physical separation of the glioma cells from the astrocytes, while allowing the transmission of secreted factors. Under this condition, astrocytes failed to protect the glioma cells from the chemotherapeutic drugs (Fig. 3A), which suggested that the protection was dependent upon physical contact.

GJC, which directly connects the cytoplasm of neighboring cells, has been shown to transmit critical secondary survival and apoptotic factors (22). Connexin 43, the major component of GJC, has been demonstrated to be expressed by astrocytes and the above-mentioned glioma cells $(33,34)$. Furthermore, functional GJC exists between astrocytes and glioma cells, as shown in in vitro and in vivo experiments (25). The present study made similar observations (data not shown). To determine whether GJC was involved in this chemoprotection, CBX, a well-documented specific inhibitor of GJC $(35,36)$ was used. As shown in Fig. 3B, the ability of the astrocytes to protect glioma cells from chemotherapeutic drugs was lost when the GJC between the astrocytes and glioma cells was inhibited.

Gene expression patterns in glioma cells are altered by astrocytes through GJC. As GJC was involved in the contact-mediated protection of glioma cells toward chemotherapeutic drugs, the present study aimed to determine the alterations in gene expression patterns in glioma cells by astrocytes through GJC. The human U87MG glioma cells were incubated for $72 \mathrm{~h}$, in the presence or absence of astrocytes, with or without the GJC inhibitor, CBX $(100 \mu \mathrm{M})$. NIH3T3 fibroblasts were used as a control. Changes in gene expression levels in the glioma cells were determined using the two sample $t$-test $(\mathrm{P}<0.001)$ in microarray analysis. By applying this procedure, co-culture with astrocytes was found to alter the expression of 3,216 genes in the U87MG glioma cells, whereas co-culture with fibroblasts altered the expression of 640 genes in the U87MG glioma cells. By applying cross comparison of the genes expressed, a total of 299 genes were found to be commonly altered by the astrocytes and fibroblasts. Therefore, these 299 genes were excluded, narrowing the list of gene, which were altered specifically by astrocytes to 2,917 (Fig. 4A and B). The present study then aimed to identify those genes that were changed via GJC. The addition of CBX $(100 \mu \mathrm{M})$ resulted in the altered expression of 1,334 genes. By comparing this list of 1,334 genes to the list of 2,917 genes, 699 genes were identified in the U87MG glioma cells, which were exclusively altered by astrocytes through GJC (Fig. 4C and D). Among these 699 genes, several genes were identified, which are known to be associated with drug resistance, anti-apoptosis and survival, including mitogen-activated protein kinase, tyrosine-protiein kinase and B cell lymphoma-2. These data demonstrated the effect of 
the brain microenvironment on glioma cells, with the unique feature of astrocytes as an example.

\section{Discussion}

As the most common type of primary brain tumor, gliomas remain one of the few types of cancer with a poor prognosis, which is primarily due to their drug resistance properties. This resistance cannot be completely attributed to the BBB, as evidence has revealed that the BBB surrounding gliomas permits leakage. The data obtained in the present study present a novel mechanism underlying this resistance, which is due to the protection from astrocytes via GJC and the upregulated expression levels of survival genes. Astrocytes maintain the homeostasis of the brain microenvironment and protect neurons from various injuries, with GJC as one of the underlying mechanisms. However, this neuroprotective feature of astrocytes is harnessed by metastatic cells in the brain (37), as has been reported previously. In the present study, it was further demonstrated that glioma cells were also able to 'exploit' this unique feature of astrocytes for their own survival benefits, to avoid apoptosis caused by chemotherapeutic drugs. In addition, the gene expression data obtained in the present study revealed the extend to which the organ microenvironment (astrocytes) affected the tumor cells (glioma) at the molecular level, and revealed genes, which were altered via GJC by neighboring cells. Taken together, these data reinforce the importance of the organ microenvironment for the behavior of tumor cells, and indicate that successful treatment of glioma in the future requires consideration of tumor cells and astrocytes, with GJC as a potential promising target.

\section{Acknowledgements}

This study was partially supported by grants from the National Nature Science Foundation (grant no. 81101664) and the Beijing Nature Science Foundation (grant no. 7132079) to Dr Qingtang Lin, and the National Science and Technology Supporting Project (grant no. 2014BAI04B00).

\section{References}

1. Wrensch M, Minn Y, Chew T, Bondy M and Berger MS Epidemiology of primary brain tumors: Current concepts and review of the literature. Neuro Oncol 4: 278-299, 2002.

2. Fisher JL, Schwartzbaum JA, Wrensch M and Wiemels JL: Epidemiology of brain tumors. Neurol Clin 25: 867-890, 2007.

3. Lévy S, Chapet S and Mazeron JJ: Management of gliomas. Cancer Radiother 18: 461-467, 2014.

4. Stupp R, Mason WP, van den Bent MJ, Weller M, Fisher B, Taphoorn MJ, Belanger $\mathrm{K}$, Brandes AA, Marosi C, Bogdahn U, et al: Radiotherapy plus concomitant and adjuvant temozolomide for glioblastoma. N Engl J Med 352: 987-996, 2005.

5. Ashmore SM, Thomas DG and Darling JL: Does P-glycoprotein play a role in clinical resistance of malignant astrocytoma? Anticancer Drugs 10: 861-872, 1999.

6. Jennings MT and Iyengar S: The molecular genetics of therapeutic resistance in malignant astrocytomas. Am J Pharmacogenomics 1: 93-99, 2001.

7. Shapiro WR and Shapiro JR: Principles of brain tumor chemotherapy. Semin Oncol 13: 56-69, 1986.

8. Mousseau M: Chemotherapy of brain tumors: Biological basis of its limited efficacy. Bull Cancer 81: 414-424, 1994 (In French).
9. Stewart DJ: A critique of the role of the blood-brain barrier in the chemotherapy of human brain tumors. J Neurooncol 20: 121-139, 1994.

10. Zhang RD, Price JE, Fujimaki T, Bucana CD and Fidler IJ: Differential permeability of the blood-brain barrier in experimental brain metastases produced by human neoplasms implanted into nude mice. Am J Pathol 141: 1115-1124, 1992.

11. Hoelzinger DB, Demuth T and Berens ME: Autocrine factors that sustain glioma invasion and paracrine biology in the brain microenvironment. J Natl Cancer Inst 99: 1583-1593, 2007.

12. Fidler IJ: The pathogenesis of cancer metastasis: The 'seed and soil' hypothesis revisited. Nat Rev Cancer 3: 453-458, 2003.

13. Abbott NJ, Rönnbäck L and Hansson E: Astrocyte-endothelial interactions at the blood-brain barrier. Nat Rev Neurosci 7: 41-53, 2006.

14. Fields RD and Stevens-Graham B: New insights into neuron-glia communication. Science 298: 556-562, 2002.

15. Bullock TH, Bennett MV, Johnston D, Josephson R, Marder E and Fields RD: Neuroscience: The neuron doctrine, redux. Science 310: 791-793, 2005.

16. Miller G: Neuroscience: The dark side of glia. Science 308: 778-781, 2005.

17. Allen NJ and Barres BA: Neuroscience: Glia-more than just brain glue. Nature 457: 675-677, 2009.

18. Sofroniew MV: Reactive astrocytes in neural repair and protection. Neuroscientist 11: 400-407, 2005.

19. Crooks DA, Scholtz CL, Vowles G, Greenwald S and Evans S: The glial reaction in closed head injuries. Neuropathol Appl Neurobiol 17: 407-411, 1991.

20. Mahesh VB, Dhandapani KM and Brann DW: Role of astrocytes in reproduction and neuroprotection. Mol Cell Endocrinol 246: $1-9,2006$

21. Chen LW, Yung KL and Chan YS: Reactive astrocytes as potential manipulation targets in novel cell replacement therapy of Parkinson's disease. Curr Drug Targets 6: 821-833, 2005.

22. Krysko DV, Leybaert L, Vandenabeele P and D'Herde K: Gap junctions and the propagation of cell survival and cell death signals. Apoptosis 10: 459-469, 2005.

23. Lin JH, Weigel $H$, Cotrina ML, Liu S, Bueno $E$, Hansen AJ, Hansen TW, Goldman S and Nedergaard M: Gap-junction-mediated propagation and amplification of cell injury. Nat Neurosci 1: 494-500, 1998.

24. Rouach N, Avignone E, Même W, Koulakoff A, Venance L, Blomstrand F and Giaume C: Gap junctions and connexin expression in the normal and pathological central nervous system. Biol Cell 94: 457-475, 2002.

25. Zhang W, Couldwell WT, Simard MF, Song H, Lin JH and Nedergaard M: Direct gap junction communication between malignant glioma cells and astrocytes. Cancer Res 59: 1994-2003, 1999.

26. Zhou B, Bu G, Zhou Y, Zhao Y, Li W and Li M: Knockdown of CDC2 expression inhibits proliferation, enhances apoptosis and increases chemosensitivity to temozolomide in glioblastoma cells. Med Oncol 32: 378, 2015.

27. Chai KM, Wang CY, Liaw HJ, Fang KM, Yang CS and Tzeng SF: Downregulation of BRCA1-BRCA2-containing complex subunit 3 sensitizes glioma cells to temozolomide. Oncotarget 5: 10901-10915, 2014

28. Langley RR, Fan D, Guo L, Zhang C, Lin Q, Brantley EC, McCarty JH and Fidler IJ: Generation of an immortalized astrocyte cell line from $\mathrm{H}-2 \mathrm{~Kb}$-tsA58 mice to study the role of astrocytes in brain metastasis. Int J Oncol 35: 665-672,2009.

29. Grossman R, Brastianos H, Blakeley JO, Mangraviti A, Lal B, Zadnik P, Hwang L, Wicks RT, Goodwin RC, Brem H and Tyler B: Combination of anti-VEGF therapy and temozolomide in two experimental human glioma models. J Neurooncol 116: 59-65, 2014.

30. Peng Q, Moan J, Ma LW and Nesland JM: Uptake, localization and photodynamic effect of meso-tetra (hydroxyphenyl) porphine and its corresponding chlorin in normal and tumor tissues of mice bearing mammary carcinoma. Cancer Res 55: 2620-2626, 1995.

31. Weihua Z, Tsan R, Huang WC, Wu Q, Chiu CH, Fidler IJ and Hung MC: Survival of cancer cells is maintained by EGFR independent of its kinase activity. Cancer Cell 13: 385-393, 2008. 
32. Bolstad BM,Irizarry RA, Astrand M and Speed TP: A comparison of normalization methods for high density oligonucleotide array data based on variance and bias. Bioinformatics 19: 185-193, 2003.

33. Hinkerohe D, Wolfkühler D, Haghikia A, Meier C, Faustmann PM and Schlegel U: Dexamethasone differentially regulates functional membrane properties in glioma cell lines and primary astrocytes in vitro. J Neurooncol 103: 479-489, 2011.

34. Zhang B, Feng X, Wang J, Xu X, Liu H and Lin N: Adenovirus-mediated delivery of bFGF small interfering RNA increases levels of connexin 43 in the glioma cell line, U251. J Exp Clin Cancer Res 29: 3, 2010.
35. Goldberg GS, Moreno AP, Bechberger JF, Hearn SS, Shivers RR, MacPhee DJ, Zhang YC and Naus CC: Evidence that disruption of connexin particle arrangements in gap junction plaques is associated with inhibition of gap junctional communication by a glycyrrhetinic acid derivative. Exp Cell Res 222: 48-53, 1996.

36. Guan X, Wilson S, Schlender KK and Ruch RJ: Gap-junction disassembly and connexin 43 dephosphorylation induced by 18 beta-glycyrrhetinic acid. Mol Carcinog 16: 157-164, 1996.

37. Fidler IJ, Balasubramanian K, Lin Q, Kim SW and Kim SJ: The brain microenvironment and cancer metastasis. Mol Cells 30: 93-98, 2010. 